\title{
DIRECT OBSERVATIONS IN THE DUSK HOURS OF THE CHARACTERISTICS OF THE STORM-TIME RING CURRENT PARTICLES DURING THE BEGINNING OF MAGNETIC STORMS
}

\section{PAUL H. SMITH}

R. A. HOFFMAN

(NASA-TM-X-70430) DIRECT OBSERVATIONS IN

\section{AUGUST 1973}




\title{
DIRECT OBSERVATIONS IN THE DUSK HOURS OF THE CHARACTERISTICS OF THE STORM-TIME RING CURRENT PARTICLES DURING THE BEGINNING OF MAGNETIC STORMS
}

\author{
By \\ Paul H. Smith \\ R. A. Hoffman \\ Laboratory for Space Physics \\ NASA-Goddard Space Flight Center \\ Greenbe1t, Maryland 20771
}

August 1973 


\section{ABSTRACT}

The characteristic features of the initial enhancement of the storm-time ring current particles in the evening hours are consistent with flow patterns resulting from a combination of inward convection, gradient drift and corotation which carries plasma sheet protons into low L-values near midnight and the higher-energy proton component into the plasmasphere and through the evening hours. Data from four magnetic storms during the early 1 ife of $\mathrm{S}^{3}-\mathrm{A}$ (Explorer 45) when the local-time of apogee was in the afternoon and evening hours show that protons with lower-magnetic moments penetrate deeper into the magnetosphere until a lower limit, determined by the corotation and gradient drift forces, is reached. Such particle motions produce the stable energy-dependent inner boundary of the ring current protons inside the plasmapause in the dusk sector and also provide the mechanism for energy injection into the ring current region. From the analyses of the pitch-angle distributions it is evident that charge exchange and wave-particle interactions are not the dominant causes of this inner boundary. Observations at altitudes just beyond the measured plasmapause of plasma sheet protons and electrons are also consistent with the resulting flow patterns. 


\section{INTRODUCTION}

While it is now generally accepted that the source of the ring current protons associated with main phase magnetic storms is the convection of plasma sheet protons into low L-values (Axford, 1969; Vasyliunas, 1972 ; and Nishida and Obayashi, 1972) there have been almost no direct experimental measurements of the characteristics of these storm-time ring current protons which would substantiate this concept.

In this analysis we demonstrate that the characteristic features of the initial enhancement of the storm-time ring current protons in the evening hours are consistent with flow patterns resulting from a combination of inward convection, gradient drift, and corotation, which carries plasma sheet protons into low L-values near midnight and the higher-energy proton component into the plasmasphere and through the evening hours.

Data for this analysis were obtained from the $S^{3}-A$ (Explorer 45) satellite, launched on November 15, 1971 into an elliptical orbit having an apogee of $5.24 \mathrm{R}_{\mathrm{E}}$, an inclination of $3.6^{\circ}$, and period of 7.8 hours (Longanecker and Hoffman, 1973).

Previous results from $S^{3}-A$ have shown that during main phase magnetic storms the storm-time ring current is due to an enhancement of the proton energy density in the energy range 10 to $100 \mathrm{keV}$ (Smith and Hoffman, 1972; Smith and Hoffman, 1973).

\section{PARAMETERS OF THE MAGNETIC STORMS}

For the study of the enhancement of ring current protons we have selected four magnetic storms during the early life of the satellite when the apogee was in the afternoon and evening hours. Some characteristic parameters pertaining to the storms and the satellite orbit numbers during the development of each main phase are listed in Table 1. 


\section{CHARACTERISTIC FEATURES OF RING CURRENT ENHANCEMENTS}

The characteristic features in the proton and electron distributions which have been observed in the evening hours during the development of the main phase for each storm are illustrated in Figure 1 . We wili show that these features provide a measure of the depth of penetration of the plasma sheet both in the evening and night hours.

Proton and electron energy spectrograms for particle fluxes with near $90^{\circ}$ pitch-angles are shown for data from a two hour period from each of two storms. In both proton spectrograms the re exists a "nose" structure beginning at lower L-values with a flux increase in the energy range 15 to $20 \mathrm{keV}$ (in Orbit 314 at $0943 \mathrm{U} . \mathrm{T}$. and in Orbit 350 at 2326 U.T.). The flux increase spreads to both higher and lower energies at larger L-values and remains at a high intensity beyond $\mathrm{L}=5.3$. This nose structure has been observed during the development of the main phase for each of the four magnetic storms we have studied. It always extends into the plasmapause and is observed near dusk in the equatorial plane. A second noticeable feature in the spectrograms is a large rapid flux increase in the 1 - to $50-\mathrm{keV}$ electrons at larger radial distances. An increase of 1 - to $2-\mathrm{keV}$ protons occurs simultaneously with the increase in the lowest-energy electrons.

Several questions immediately arise upon considering these observations. 1. Is the nose structure a spatial or temporal phenomenon, i.e., is the satellite passing through these characteristic patterns which have considerable local-time extent and are relatively stationary throughout the development of the main phase, or is the satellite being enveloped for a time by changing plasma distributions which are 
relatively independent of the L-values of the measurements? 2 . What role, if any, does the plasmasphere play in the formation of the nose? 3. Why is the center energy of the nose around 15 to $20 \mathrm{keV}$, and why is the flux increase of the higher- and also lower-energy protons observed at larger L-values? 4. What accounts for the simultaneous observation of the flux increase of the very low-energy electrons and protons?

\section{SPATIAL NATURE OF NOSE EVENTS}

There are several pieces of evidence which indicate that the nose structures are spatial. For each of the four magnetic storms the location of the nose structure and the satellite positions at the time of the storm sudden commencement and onset of the main phase are indicated in Figure 2. The time interval $(\Delta t)$ between the onset of the main phase, defined as the average time when the slope of $\Delta H$ became negative on several mid-and low-latitude magnetograms, and the observation of the nose is also listed. The time intervals range from 10 minutes to over 400 minutes. In the first three storms the nose is observed on the outbound leg of the orbit at or just before dusk, whereas, in the fourth storm it is observed on the inbound leg somewhat after dusk. It appears that the nose structure is a rather stable feature in the dusk quadrant during the development of the main phase owing to the fact that it is observed at arbitary times during the development of the main phase, over a range of local times, and irrespective of whether the satellite is inbound or outbound. We can envision such a persistent feature existing in a region of local time only as a spatial structure. 
An argument against the nose being a temporal phenomenon is the fact that its energy structure cannot be explained by the impulsive injection of a cloud of protons near midnight, with the spacecraft observing merely the dispersion in the time of arrival for protons of various energies due to energy-dependent gradient drift (DeForest and McIlwain, 1971). In Figure 3 the Universal Time at which the large fl ux gradient was observed is plotted for each of the measured energy bands for the nose structure of Orbit 314. The calculated longitudinal drift times (Lew, 1961) for protons at these energies, injected near midnight and gradient drifting around to the point of the satellite observation, are shown as the solid line. This line is normalized to $13.5 \mathrm{keV}$ to correspond to the earliest proton flux increase. As expected, this simple model cannot explain the later arrival of the higher-energy protons, and it also fails to predict quantitatively the delay times with which the lower-energy protons were observed.

Therefore, on the basis of the above arguments, we believe the nose is a spatial structure extending over a number of hours in local time.

\section{EFFECTS OF THE PLASMAPAUSE}

Having demonstrated that the nose structure is most probably a stable energy-dependent inner boundary of the storm-time ring current protons, we next investigate whether it can be explained as an interaction of the protons with the plasmasphere. We note in Figure 2 that the point of the nose was always observed inside the plasmapause. Two 
types of interactions will be considered: charge exchange and resonance interactions with waves.

Cornwal1 et a1. (1970) point out that charge exchange loss rates should not exhibit an abrupt change across the plasmapause, because the neutral particles, which are unaffected by convection electric fields and drifts, should have a smooth density profile across the plasmapause. It should also be noted that charge exchange lifetimes for protons with $90^{\circ}$ pitch-angles are about one day and are quite independent of energy from 5 to $30 \mathrm{keV}$, and that the lifetimes rapidly increase for higher-energy protons (Liemohn, 1961). In comparison, the gradient drift times from midnight to dusk for protons with nose energies (15 $20 \mathrm{keV}$ ) are about three hours. Therefore, the effect of charge exchange losses on the protons with nose energies which are assumed to be drifting from midnight through the dusk hours and into the plasmasphere, would be to produce smooth but slowly decreasing proton fluxes across the plasmapause. The relative flux decreases also should be similar for protons up to about $30 \mathrm{keV}$, with the higher-energy protons decreasing less rapidly in the gradient region of the lower-energy protons.

The observations, however, are not consistent with charge exchange losses. A more detailed set of data, which is typical of each of the storms we have studied, is shown in Figure 4. The center pane 1 repeats the proton energy spectrogram from Figure 1, while the top panel contains the actual flux profiles for $90^{\circ}$ pitch-angle protons for several bands of energy whose widths are about $\pm 15 \%$ of the center energies 1isted. Note that the flux profiles are highly energy dependent, with 
the $42.8 \mathrm{keV}$ proton profile decreasing at largest $\mathrm{L}$ rather than at smallest $L$ as would be expected from charge exchange losses. In addition, the changes of intensity are too abrupt to be explained by a loss process which slowly varies with L.

Further evidence against a simple loss process for explaining any of the nose characteristics comes from a consideration of the pitchangle distributions, shown in the bottom panel for five places in the nose structure. Since the relative charge exchange lifetimes as a function of pitch-angle only depend upon the neutral density variation along a line of force, the pitch-angle distributions should be similar at a given L-value (Liemohn, 1961). In contrast it is seen that the pitch-angle distribution at the nose energy of $13.5 \mathrm{keV}$ is steepest throughout most of the structure.

Next we consider whether the nose structure can be due to pitchangle scattering, possibly from some process like the ion-cyclotron instability. While the flux profiles in the top panel of Figure 4 are reminiscent of those published by Williams et a1. (1973) from the December 1971 storm and interpreted as possibly resulting from an ioncyclotron instability, the energies in the nose structure are predominantly below those they considered as being involved in this process. We also conclude that the protons of nose energies are not involved in this type of instability from a further consideration of the pitch-angle distributions. Lyons et al. (1972) showed that a resonance process between waves and the parallel component of the momentum 
of the particles results in pitch-angle distributions which have "shoulders", i.e., the near $90^{\circ}$ pitch-angle particles will be basically unaffected, and the gradient of the pitch-angle distribution will have a secondary minimum at some intermediate pitch-angle. The lower-energy particles will have this secondary minimum nearest to $90^{\circ}$. Finally, the lowest-energy protons will become involved in the resonance at the lowest L-value, since the resonance energy is inversely proportional to the plasma density (Kenne1 and Petschek, 1966).

In contrast to the results expected from the theory, the pitchangle distributions in Figure 4 show a precipitous decrease in the $90^{\circ}$ fluxes at energies both above and below the nose energy, while the flux at the nose energy remains constant from 0950 to 1010 U.T. No shoulder structures are consistently evident in the distributions, and the protons with off $-90^{\circ}$ pitch-angles at the lowest energy $(6 \mathrm{keV}$ in the 1000 U.T. distribution) become involved at the highest I-value instead of at the lowest L-values.

Returning to Figure 1, we further note that for Orbit 350 a portion of the nose structure lies outside the local plasmapause. This provides additional evidence that interactions of the protons with the plasmasphere do not play a significant role in the formation of the nose structure.

EFFECTS OF CONVECTION AND DRIFT ON ENERGY DEPENDENT BOUNDARIES

We next look to convection theory to explain the existence of the nose structure, the intensification of protons at larger L-values, and 
the sudden enhancement of electron and 1-keV proton fluxes outside the p1asmapause.

The effects of the transport of the ions and electrons by convection and magnetic drift are examined in order to explain the deeper penetration of the lower-energy protons into the magnetosphere, i.e., the slope of the upper portion of the nose. From the convection analyses of Chen (1970), Jaggi and Wolf (1973), and Chen (private communication, 1973), it is concluded that the minimum geocentric distance to which convection fields can bring ions to the dusk meridian is roughly proportional to $\mu^{1 / 3}$, where $\mu$ is the proton magnetic moment. Thus the lower-magnetic-moment protons penetrate deeper into the magnetosphere. Chen assumed a dawn-dusk electric field across the entire magnetosphere and calculated the plasma flow patterns for various magnetic moments without considering the perturbations on the electric field caused by the plasma sheet particles themselves. Jaggi and Wolf, on the other hand, included electrostatic shielding effects earthward of the Alfvén layer in calculating the closest approach for the plasma sheet, which was assumed to be composed of ions of a single magnetic moment.

Chen's analysis also showed, even with the simplifying assumption, a forbidden region on the dusk side for protons with small magnetic moments $(\mu=1.25 \mathrm{eV} /$ gamma $)$. The forbidden region, which rapidly shrank for higher magnetic moments, resulted from the corotation of the low-moment ions to the dawn side. Jaggi and Wolf demonstrated similar forbidden regions on the dusk side. 
Thus the nose structure can be explained by the fact that the lowermoment ions would penetrate deeper into the magnetosphere until a critical value of $\mu$ is reached. This critical value is determined by equal opposing gradient drift and corotational forces. Ions with moments smaller than the critical value would drift eastward thereby producing the lower-energy edge of the nose structure due to their absence in the dusk sector.

Having considered the transport of ions to allowed or forbidden regions, we will now discuss the effects of the energization of the ions due to the cross-L motion resulting from the convection, which also effects the intensities of the ions. First it is assumed that the particle source of the ring current protons is a portion of the quiet-time plasma sheet, where the density of ions for each magnetic moment is reasonably constant in the region of the plasma sheet of interest. Idea11y one would want to follow an element in phase space as the ions in this element drift from their origin into low L-values. According to Liouville's theorem the density in phase space of these ions would remain constant if there were no loss processes. As applied to our problem, it is not possible to follow such an element in phase space, but only to investigate the implications of Liouville's theorem. Under the assumption of a reasonably constant density in the region of the source, and if no loss processes occur during the transport of these ions to the L-values and local-time of the measurements, then the density in phase space over the allowed region of real space should be found to be constant. Ions with different magnetic moments 
will have different allowed regions and different values of the density. The density in phase space is proportional to the flux divided by the energy, J/E.

In order to show the features in the data which are expected from the effects of the transport and energization, the phase space density profiles for magnetic moments from 10 to $400 \mathrm{eV} /$ gamma for Orbit 314 outbound are plotted in Figure 5. Clearly the 40-eV/gamma protons penetrate to lowest L-values, with nearly constant phase space density, and the densities of the higher-magnetic-moment protons begin decreasing at larger L-values, as the calculations predict. However for these higher-moment protons, we find a much less sensitive dependence than is predicted for the minimum L-value to which a given $\mu$ particle can penetrate. While the lower than $40-\mathrm{eV} /$ gamma protons would penetrate to still lower L-values probably near midnight, they would not drift to the 1oca1-time of the measurements because of the dominating corotation force on these particles. At L-values higher than the respective cut-offs, the densities in phase space are quite constant until magnetic moments of $200 \mathrm{eV} /$ gamma are reached. At an L of 5, this corresponds to an energy of about $45 \mathrm{keV}$, which then must be the upper limit to the protons involved in the storm-time ring current at this stage of the storm.

The constancy of the phase space density a1so implies that the protons at deeper L-values have been more extensively energized, since to keep $\mu$ constant the perpendicular energy must be larger at the larger magnetic field values. If the spectrum is monotonically decreasing with 
increasing $\mu$, the effects of this energization would be an increasing flux at a given energy with decreasing L. The 13.5-and 25.6-keV flux contours in the upper portion of Figure 4 illustrate this property.

The constancy of the density probably eliminates the idea of an in situ acceleration of ring current protons. For such a mechanism one would expect a density maximum at the L-value of the most efficient or strongest acceleration.

Finally, we can account for the existence of the high fluxes of both protons with energies less than $2 \mathrm{keV}$ and electrons just outside the plasmapause (Figure 1). Because very low-energy particles of each charge are observed simultaneously, one concludes that these particles are moving like zero magnetic-moment particles, or nearly along equipotentials. Jaggi and Wolf (1973) in their model show zero-energy particles drifting to within $5.2 \mathrm{R}_{\mathrm{E}}$ at dusk six hours after the ion sheet started in from the tail (see their Figure 8). These particles must therefore comprise the inward-most extension of the plasma sheet at this local time.

\section{INTERPRETATION}

A schematic of the trajectories of particles for several magnetic moments is shown in Figure 6 assuming a cross-tail electric field. Al1 charged particles, independent of energy, convect inward until gradient drift and corotation become important. The electrons and very lowenergy protons follow eastward trajectories through the dawn hours under the influence of corotation. The higher-energy protons become dominated by gradient drift and follow westward trajectories into the 
region of the satel1ite observations. Protons with magnetic moments of $10 \mathrm{eV} /$ gamma have equal opposing forces from corotation and gradient drift at $\mathrm{L}=3$. In the dusk hours the observations show that the lowermagnetic-moment protons penetrate to lower L-values until a critical value of the magnetic moment is reached. Therefore, the plasma flow patterns, taking into account convection, corotation and gradient drift, which Chen and Jaggi and Wolf have calculated, explain in a qualitative sense the nose structure in the dusk hours. We are not yet ready to provide a quantitative comparison between the observations and the calculations.

Such flow patterns also explain why noses occur inside the plasmapause. The protons have drifted from near midnight into the bulge region of the plasmasphere in the dusk hours without undergoing any significant losses (Figure 7).

Thus, in reality, we have two local time measurements of the closest distance of convection associated with the enhancement of the ring current during magnetic storms (Figure 7): one is a local measurement near dusk, consisting of the direct observation of near zero magnetic-moment particles a few tenths of an earth radius outside the plasmapause; the other is a remote measurement, provided by the nose structure, which reflects the consequences of convection of the inner edge of the plasma sheet near midnight. 


\section{ACKNOWLEDGEMENTS}

We acknowledge the contributions of Drs. T. A. Fritz, D. J. Williams, N. C. Maynard and L. J. Cahil1, Jr. in providing the experiment complement of $\mathrm{S}^{3}-\mathrm{A}$ from which data for this study were used. We wish to especially thank Drs. J. L. Burch, A. J. Chen, L. R. Lyons, D. J. Williams, T. A. Fritz, and D. P. Stern for very valuable discussions. We are grateful to Christine Gloeckler for developing the computer programs which were used in this analysis. 


\section{REFERENCE}

Axford, W. I., Magnetospheric convection, Rev. Geophys., I, 421, 1969. Chen, A. J., Penetration of low-energy protons deep into the magnetosphere, J. Geophys. Res., 75, 2458-2467, 1970 .

Cornwa11, J. M., F. V. Coroniti, and R. M. Thorne, Turbulent loss of ring current protons, J. Geophys. Res., 75, 4699-4709, 1970.

DeForest, S. E., and C. E. McIlwain, Plasma clouds in the magnetosphere, J. Geophys. Res., 76, 3587-3611, 1971 .

Jaggi, R. K., and R. A. Wolf, Self-consistent calculation of the motion of a sheet of ions in the magnetosphere, J. Geophys. Res., $\underline{78}$, $2852-2866,1973$.

Kennel, C. F., and H. E. Petschek, Limit on stably trapped particle fluxes, J. Geophys. Res., 71, 1-28, 1966.

Lew, J. S., Drift rate in a dipole field, J. Geophys. Res., 66, 2681$2685,1961$.

Liemohn, H., The lifetimes of radiation belt protons with energies between $1 \mathrm{keV}$ and $1 \mathrm{MeV}$, J. Geophys. Res., 66, 3593-3595, 1961.

Longanecker, G. W., and R. A. Hoffman, $S^{3}-A$ spacecraft and experiment description, J. Geophys. Res., 78, Aug. 1973.

Lyons, L. R., R. M. Thorne, and C. F. Kennel, Pitch-angle diffusion of radiation belt electrons within the plasmasphere, J. Geophys. Res., 77, $3455-3474,1972$

Maynard, N. C., and D. P. Cauffman, Double-floating probe measurements

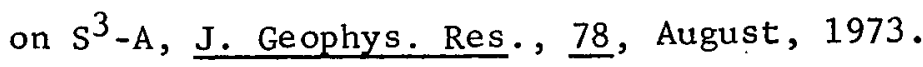


Nishida, A., and T. Obayashi, Magnetosphere convection, in Critical Problems of Magnetospheric Physics, Pruceedings of the Joint COSPAR/ IAGA/URSI Symposium, Madrid, Spain, May 11-13, 1972, Edited by

E. R. Dyer, IUCSTP Secretariat, \% National Academy of Sciences, Washington, D. C., p. 179, 1972 .

Smith, P. H., and R. A. Hoffman, Storm-time ring current distributions for $5 \mathrm{keV}$ to $872 \mathrm{keV}$ protons, Trans. Am. Geophys. Union 53, 1088, 1972 .

Smith, P. H., and R. A. Hoffman, Ring current particle distributions during the magnetic storms of December 16-18, 1971, J. Geophys. Res., $\underline{78}$, August, 1973.

Vasyliunas, V. M., The interrelationship of magnetospheric processes, Earth's Magnetospheric Processes, ed. B. M. McCormac, D. Reidel Pub., Dordrecht-Holland, pp. 29-38, 1972.

Williams, D. J., T. A. Fritz, and A. Konradi, Observations of proton spectra $(1.0 \leq \mathrm{Ep} \leq 300 \mathrm{keV})$ and fluxes at the plasmapause, $\mathrm{J}$. Geophys. Res., 78, August, 1973. 
TABLE 1. Parameters of the Magnetic Storms

\begin{tabular}{|c|c|c|c|c|}
\hline DATE & S.C. (U.T.)* & $\begin{array}{l}\text { ONSET OF } \\
\text { MAIN PHASE } \\
\text { (U.T.) }\end{array}$ & $\begin{array}{l}\text { APPROX. } \\
\text { MAGNITUDE } \\
\text { OF M. P. }\end{array}$ & $\begin{array}{l}S^{3}-A \\
\text { ORBIT } \\
\text { NUMBER }\end{array}$ \\
\hline Dec. 17,1971 & 1418 & 1500 & $225 \gamma$ & 101 \\
\hline Feb. 24,1972 & 0642 & 0800 & $140 \gamma$ & 314 \\
\hline Mar. 6, 1972 & 2108 & 2315 & $165 \gamma$ & 350 \\
\hline June 17,1972 & 1312 & 2040 & $260 \gamma$ & 671 \\
\hline
\end{tabular}

*Solar-Geophysical Data, 330 Part 1, pp. $96 \& 97$, Feb. $1972 ; 332$ Part 1, pp. 104 \& 105, April 1972; 333 Part 1, pp. $102 \& 103$, May 1972; 336 Part 1, pp. $120 \& 121$, Aug. 1972; U. S. Department of Commerce, Boulder, Colorado 80302 . 


\section{FIGURE CAPTIONS}

Figure 1. Proton and electron energy spectrograms for two $\mathrm{S}^{3}-\mathrm{A}$ orbits during the development of the main phase of two magnetic storms. The grey shading is a measure of the flux (particles/ $\mathrm{cm}^{2}$-sec-ster-keV) of the equatorially mirroring particles. Black represents the most intense flux. These are unretouched computer generated plots with obvious data gaps. The plasmapause as determined by $\mathrm{s}^{3}$ instrumentation (Maynard and Cauffman, 1973) is indicated by P.P.

Figure 2. The $\mathrm{S}^{3}$-A orbit, plotted in $\mathrm{L}$ and magnetic local time coordinates during four main phase magnetic storms. The positions of the satellite at the times of the storm sudden commencements (S.SC) and the onsets of the main phases (M.P.) are indicated. During the dashed portion of the orbit the satellite was outside the measured plasmapause, indicated by the bars marked P.P. The open arrow shows the direction of orbital motion of the satellite.

Figure 3. Comparison of times of flux increases at the satellite as a function of energy with calculated times of arrival for protons injected at midnight and gradient drifted to the satellite.

Figure 4. A more detailed set of data from a nose structure during the storm of February 24, 1972. The top panel contains profiles of four energy bands of protons whose widths are about $\pm 15 \%$ around the center energies listed. The center panel contains the proton spectrogram shown in Figure 1. The lower panel 
contains pitch-angle distributions for the same four proton energy bands as the top panel at five selected positions in the nose structure. Each pitch-angle distribution consists of data from approximately $20^{\circ}$ to $160^{\circ}$.

Figure 5. Profiles proportional to phase space density (flux divided by energy) for protons with magnetic moments from 10 to $400 \mathrm{eV} /$ gamma during the development of the main phase magnetic storm on February $24,1972$.

Figure 6. Schematic of proton and electron trajectories in the equatorial plane under the influence of a cross-tail electric field, gradient drift and corotation, superimposed on the $s^{3}$-A orbit. Proton trajectories for several magnetic moments are indicated with $\mu=100$ gradient drifting faster than the lower $\mu$ 's, and $\mu=20$ penetrating deeper at dusk than the higher $\mu$ 's. At $L=3, \mu=10$ protons are balanced by corotation and gradient drift.

Figure 7. Schematic showing the region of observation of the energydependent inner boundaries of the ring current protons (nose structure) near dusk inside the plasmapause, and the high flux region (shaded) of the electrons and low-energy protons outside the plasmapause. Several trajectories for particles of $\mu=0$ and $\mu=40$ are indicated. 

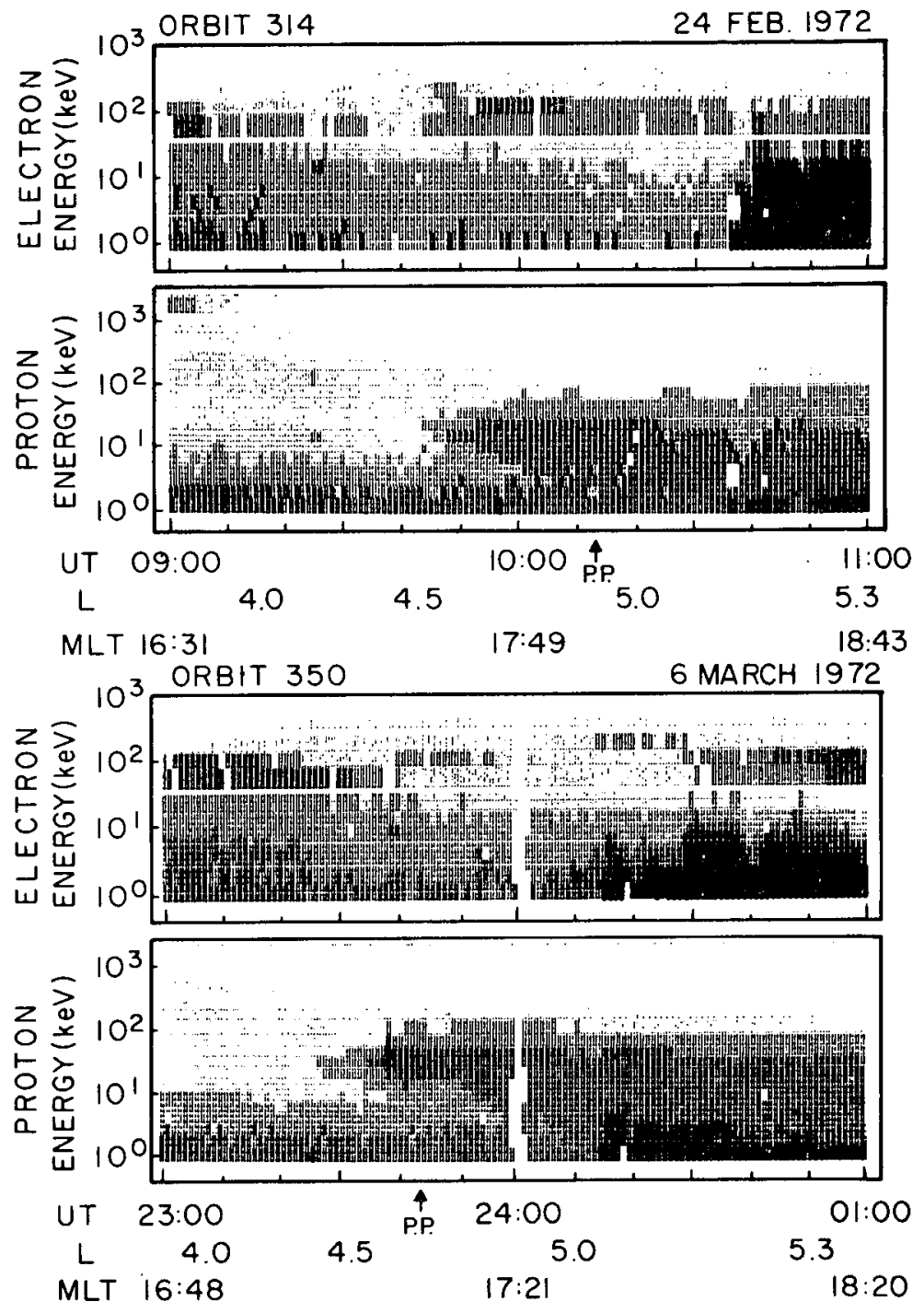

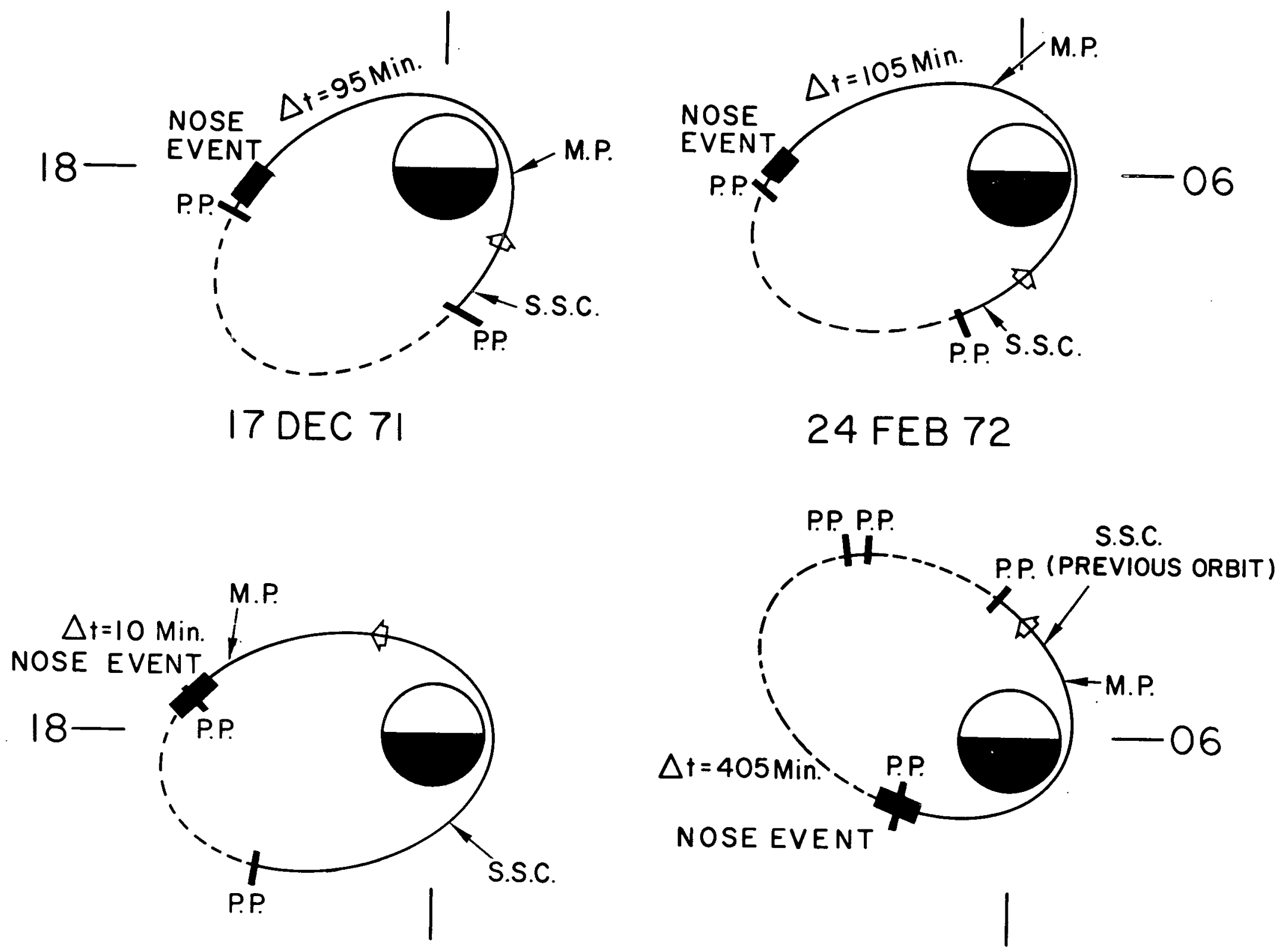

6 MAR 72

17 JUNE 72 


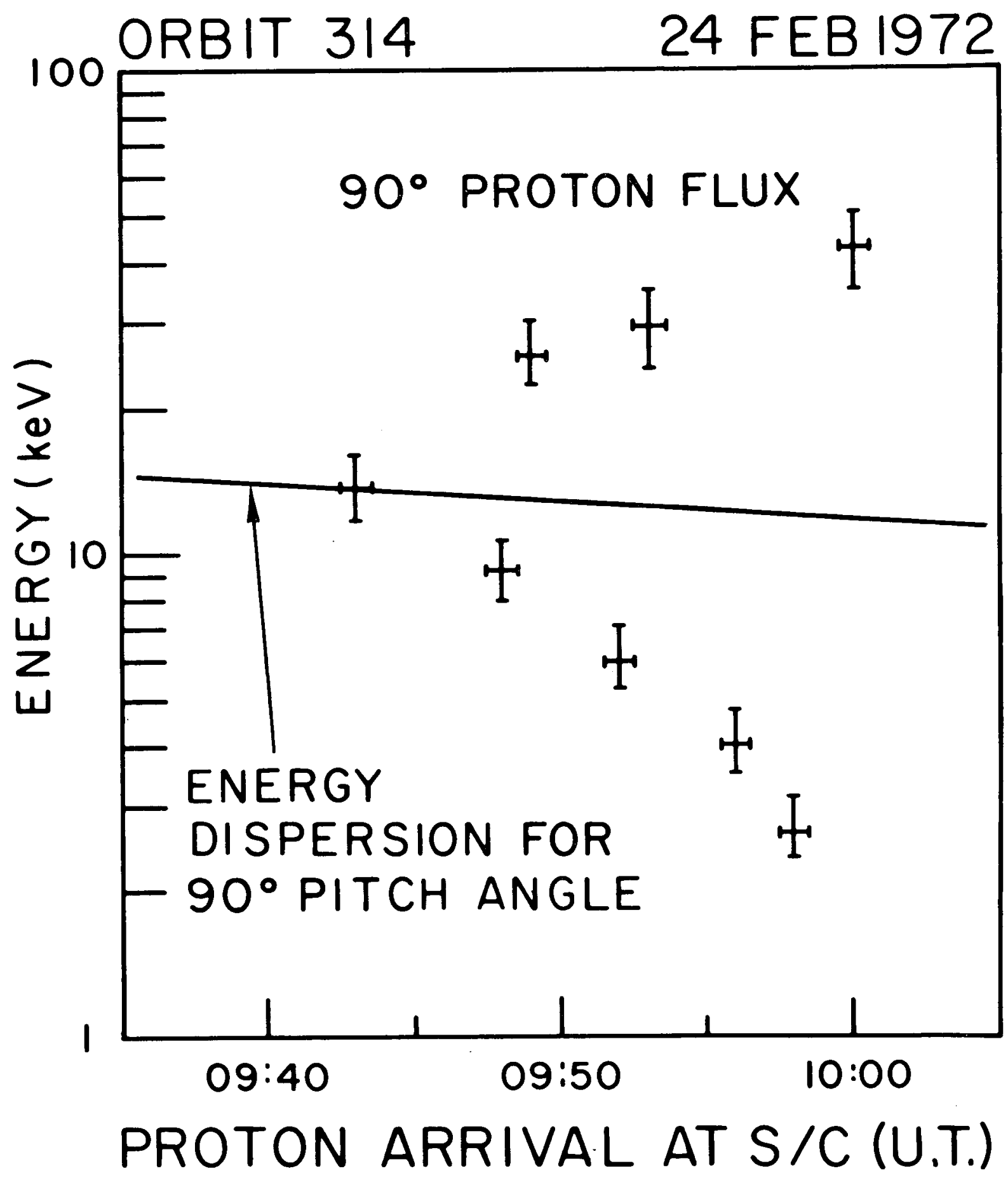



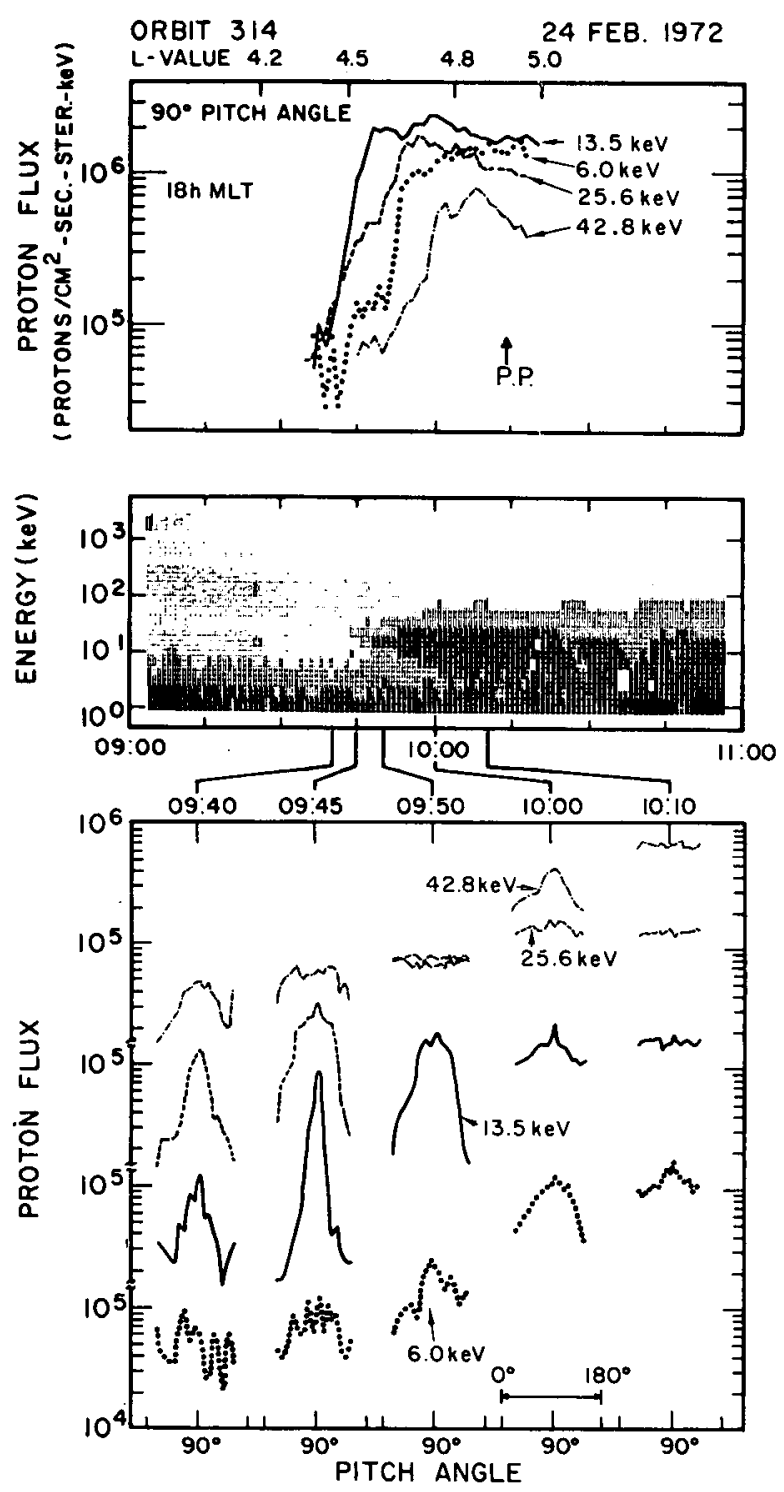


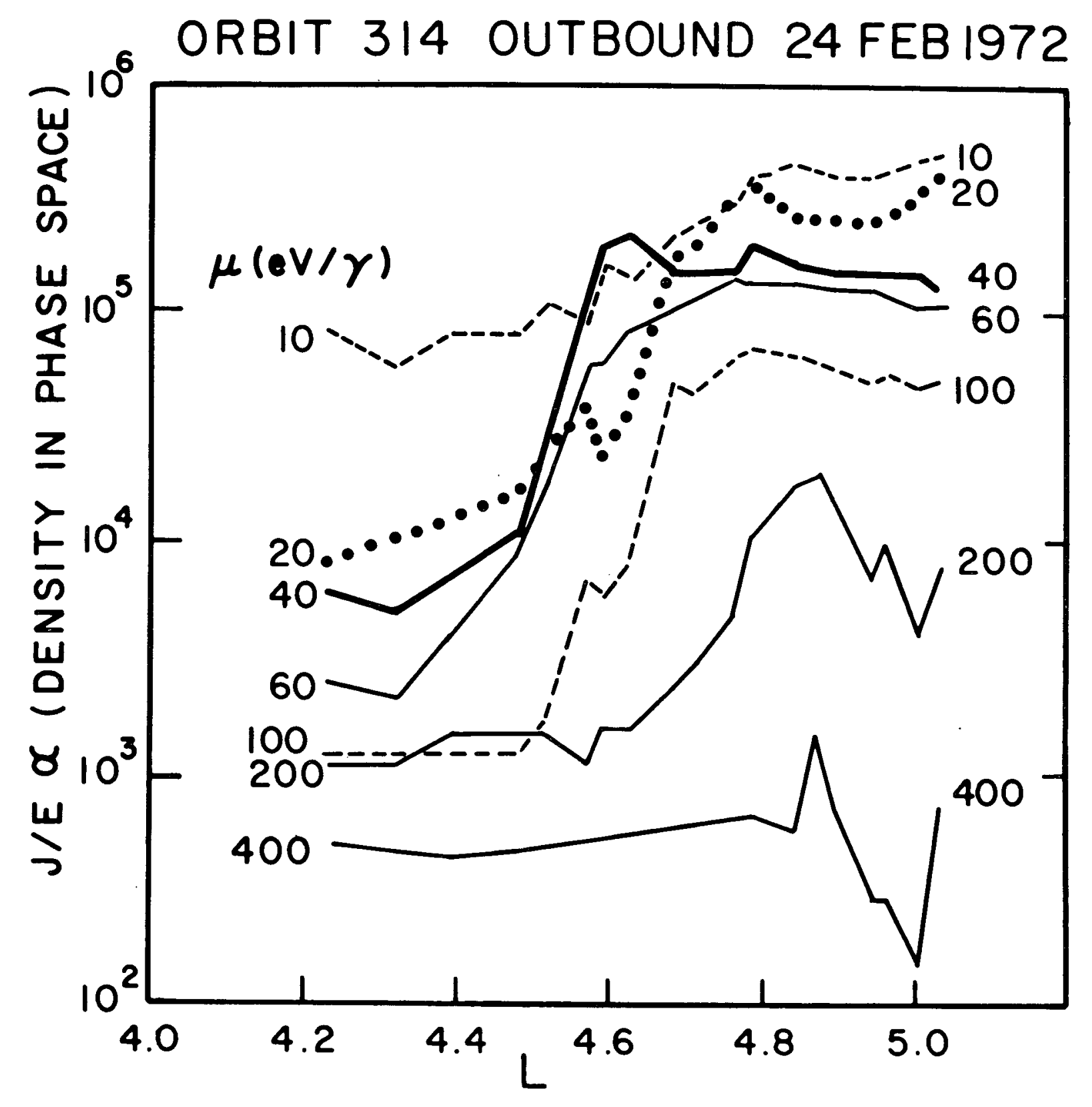




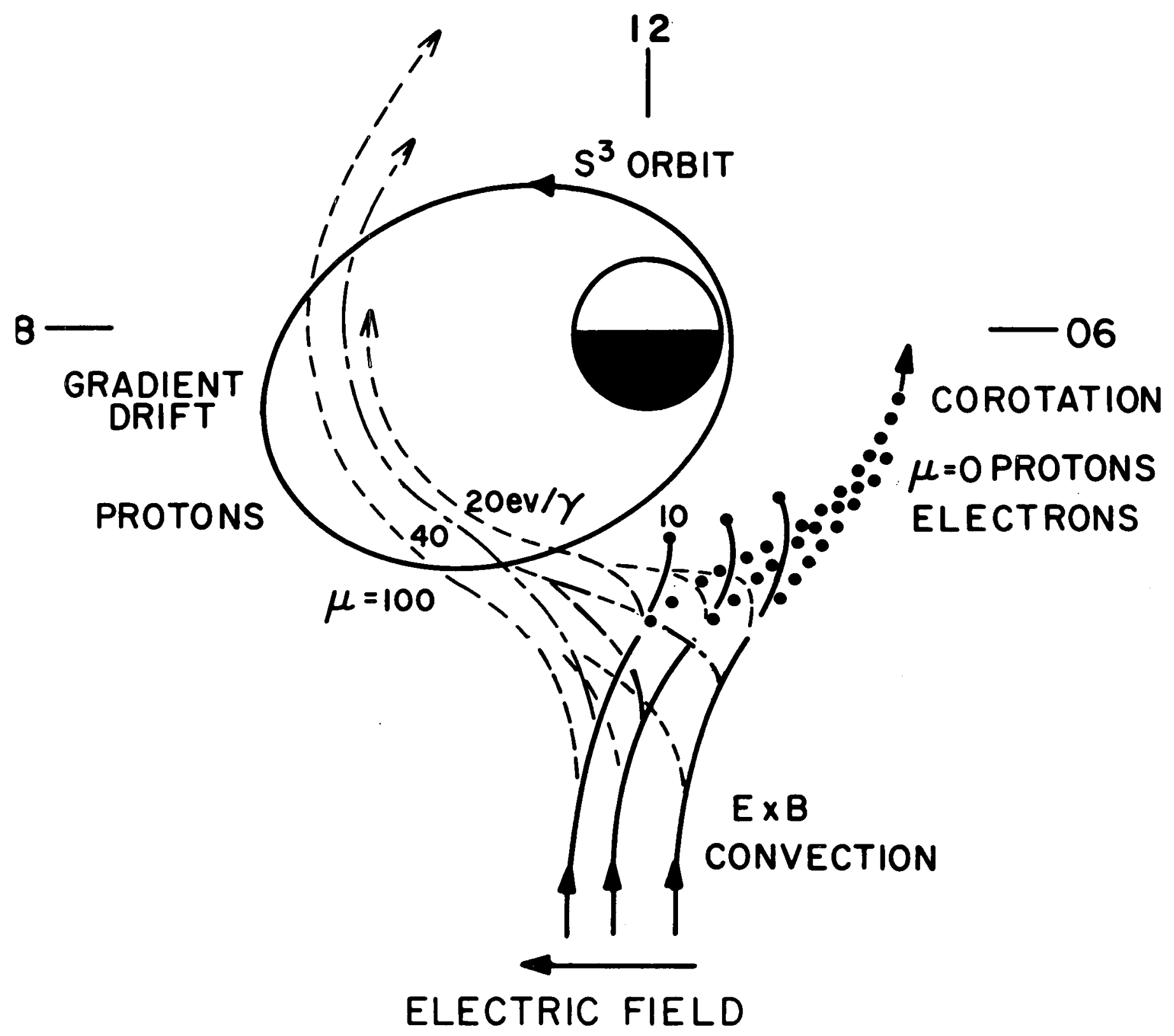




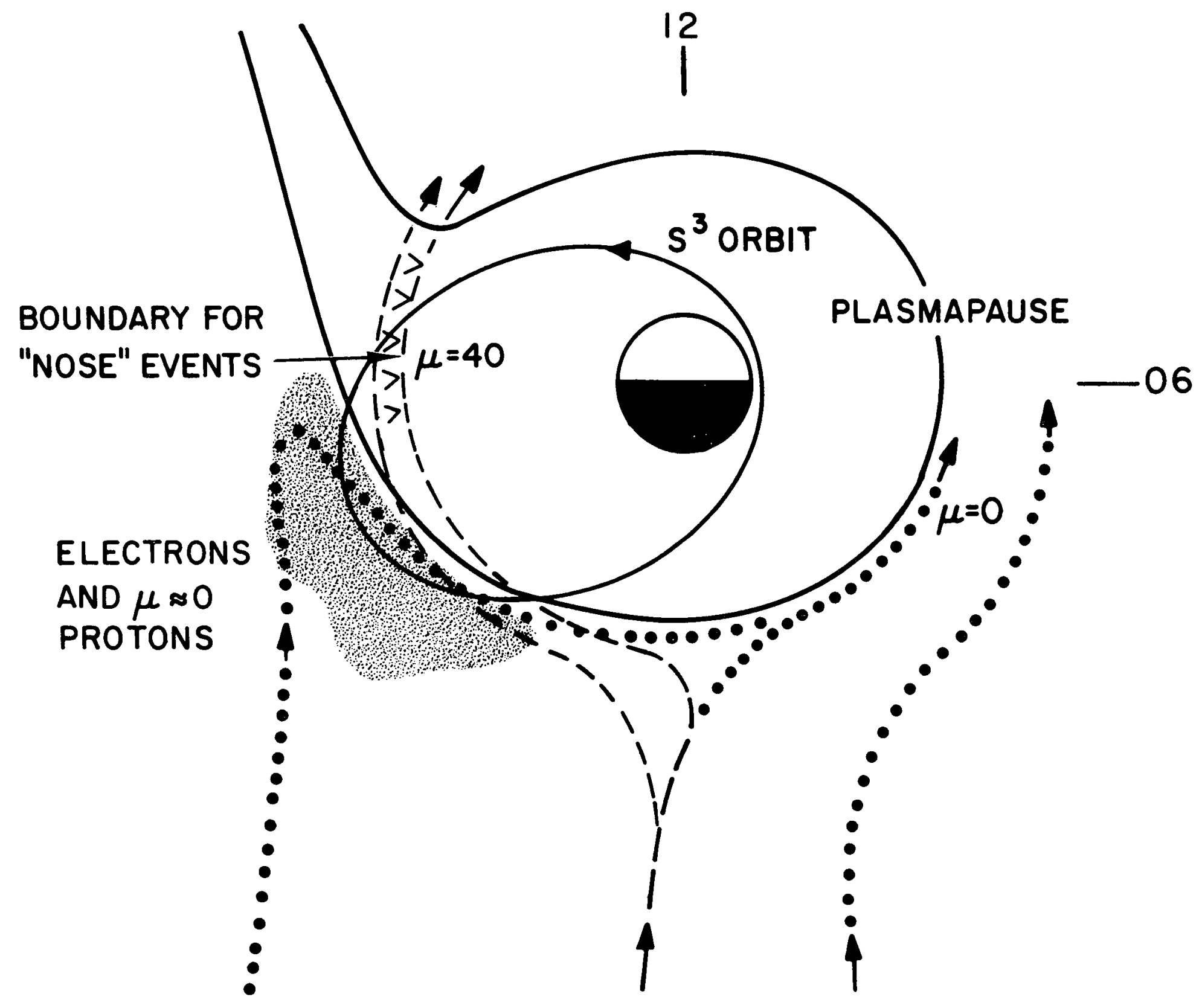

